\title{
Ultrasonography of MADSAM neuropathy: focal nerve enlargements at sites of existing and resolved conduction blocks
}

Erika Scheidl MD르 Josef Böhm $\mathrm{MD}^{2}$, Magdolna Simó MD, $\mathrm{PhD}^{1}$, Csilla Rózsa MD, $\mathrm{PhD}^{3}$, Benjamin Bereznai MD, $\mathrm{PhD}^{1}$, Tibor Kovács $\mathrm{MD}, \mathrm{PhD}^{1}$, Zsuzsanna Arányi $\mathrm{MD}, \mathrm{PhD}^{1}$

${ }^{1}$ Dept. of Neurology, Semmelweis University, Budapest, Hungary

${ }^{2}$ Neurologische Klinik, Kreiskrankenhaus Freiberg gGmbH, Freiberg, Germany

${ }^{3}$ Dept. of Neurology, Jahn Ferenc Hospital, Budapest, Hungary

Corresponding author:

Erika Scheidl MD

Dept. of Neurology, Semmelweis University, Budapest, Hungary

Balassa u. 6., Budapest, H-1083

E-mail: erika.scheidl@ chello.hu

Running title: Ultrasonography of MADSAM neuropathy 


\title{
Ultrasonography of MADSAM neuropathy: focal nerve enlargements at sites of existing and resolved conduction blocks
}

\begin{abstract}
Using the emerging technique of peripheral nerve ultrasonography, multiple focal nerve swellings corresponding to sites of existing conduction blocks have been described in demyelinating polyneuropathies. We report two cases of multifocal acquired demyelinating sensory and motor neuropathy (MADSAM). In the first, multiple focal nerve enlargements were detected by ultrasound at sites of previous conduction blocks, well after complete clinical and electrophysiological resolution. In the second case, existing proximal conduction blocks could be localized by ultrasound. Our cases highlight the importance of nerve ultrasound in identifying conduction blocks and demonstrate that ultrasonographic morphological changes may outlast functional recovery in demyelinating neuropathies.
\end{abstract}

Key words: MADSAM neuropathy, peripheral nerve ultrasound, demyelinating polyneuropathies, multifocal nerve enlargement, conduction block 


\section{Introduction}

Superficial peripheral nerves can be easily investigated by high resolution ultrasonography, allowing precise morphological analysis of nerves. Ultrasonography measurements provide useful complementary information to electrodiagnostic data. ${ }^{1}$ Sonographic diffuse or multifocal nerve enlargement were reported in various demyelinating polyneuropathies, such as chronic inflammatory demyelinating polyneuropathy (CIDP), multifocal motor neuropathy (MMN), and some hereditary polyneuropathies. ${ }^{2-6}$ Previous studies described swelling of a single hypoechoic fascicle resulting in focal enlargement of nerve diameter at sites of concurrent conduction blocks in CIDP patients. ${ }^{7,8}$

MADSAM is an immune mediated neuropathy, a variant of CIDP, which appears clinically as a chronic sensorimotor multiple mononeuropathy with multifocal conduction blocks in electrophysiological studies. ${ }^{9}$

We report the sonographic and electrophysiological findings of two patients with MADSAM neuropathy. Electrophysiological studies were performed using Nicolet Viking and Cadwell Excel EMG devices. For ultrasound examinations, a Philips HD11XE ultrasound device with a small part imaging software and a $15 \mathrm{MHz} 3 \mathrm{~cm}$ linear array transducer was used. The sonographer was blinded to clinical and electrodiagnostic data.

\section{Case report 1}

A 41-year-old male patient with an uneventful medical history presented with a sensorimotor peroneal nerve palsy on the left side in November 2008, few weeks after receiving vaccination against influenza A and B virus. Nerve conduction study of the left common 
peroneal nerve showed a conduction block at the fibular head (performed in another institute). In May 2009, a sensorimotor ulnar nerve palsy on the left side developed, while the peroneal nerve palsy still persisted. Thorough electrophysiological investigation revealed a sensorimotor conduction block of the left ulnar nerve at an atypical site, on the forearm, approximately $13 \mathrm{~cm}$ proximal to the wrist, involving over $95 \%$ of fibers (Fig. 1). The peroneal nerve conduction block was unchanged. Additional signs of demyelination were also found, such as focal demyelination of the right ulnar nerve at the elbow, and a proximal involvement of the left median nerve (increased F-wave latency). Axonal involvement was minimal. Based on the clinical presentation of sensorimotor multiple mononeuropathy and the electrophysiological findings of corresponding conduction blocks, the diagnosis of MADSAM polyneuropathy was made. Additional evaluations included genetic testing of the PMP22 gene, which was negative, excluding hereditary neuropathy with liability to pressure palsies (HNPP). Serum anti-ganglioside antibodies GM1 and GD1b were positive, supporting the immunological nature of the disease. Monoclonal gammopathy or other systemic autoantibodies were not found. Examination of the cerebrospinal fluid showed a slightly elevated protein level $(0.60 \mathrm{~g} / \mathrm{L})$, cell count and immunoassay were normal.

After oral corticosteroid treatment $(1 \mathrm{mg} / \mathrm{kg})$, the peroneal and ulnar nerve palsy resolved completely, however, radial nerve palsy on the left side developed. In March 2010, a course of plasma exchange was carried out without any improvement. After intravenous immunoglobulin treatment (at the dose of $2 \mathrm{~g} / \mathrm{kg}$ given over the course of 5 days, followed by continuous repeated doses of $0.5 \mathrm{~g} / \mathrm{kg}$ every 6 weeks), the left radial nerve palsy showed slow clinical improvement, with remaining weakness of finger extensors (May 2011), and no new symptoms developed.

Follow-up electrophysiological examination in May 2011 showed completely normal motor conduction studies (including $\mathrm{F}$ waves) of the left common peroneal and ulnar nerves. The 
sensory potential of the left ulnar nerve was of low amplitude, the peroneal sensory potential was normal. The still symptomatic left radial nerve showed marked distal sensorimotor axonal loss, which rendered the evaluation of any remaining conduction block unfeasible. Other examined nerves were normal. Ultrasound examination of the peripheral nerves was performed on the same day. An enlargement of the left radial nerve on the mid-upper-arm passing through the spiral groove was seen: on transverse scan, the cross sectional area (CSA) was significantly enlarged with a homogenous hypoechoic appearance due to loss of the normal fascicular pattern and indistinct outer margins. On longitudinal scan, an abrupt caliber change and a spindle-like swelling of this nerve segment could be seen. Similar changes were observed on the left median nerve on the upper arm, on the left ulnar nerve in the condylar groove and the distal forearm (13 cm above the styloid process of the ulna ) and on the left peroneal nerve in the distal part of the popliteal fossa (Fig. 1).

\section{Case report 2}

The 51-year-old female patient with an uneventful medical history presented with sensorimotor ulnar nerve palsy on the left side in October 2010 followed by a partial right median nerve involvement ( numbness of the index and middle finger). Six months later, sensorimotor peroneal nerve palsy on the left side developed. The patient was first evaluated in our department in June 2011. Neurological examination revealed ulnar and peroneal nerve palsies on the left side with generalized hyporeflexia and no atrophy of the involved muscles. MRI of the cervical spine showed no abnormality. Electrophysiological work-up showed a normal motor nerve conduction study of the left common peroneal nerve ( recorded from the extensor digitorum brevis muscle and the tibialis anterior muscle), and a normal sensory nerve conduction study of the left superficial peroneal nerve, but peroneal $\mathrm{F}$ waves were absent. Magnetic stimulation of the lumbar roots, recorded from the tibialis anterior muscle, showed a 
significantly increased latency of the lumbar response on the left side with increased temporal dispersion and reduced amplitude, as opposed to a normal lumbar response on the right side. This indicates that a demyelinating lesion is present on the peroneal nerve proximal to the most proximal site accessed by electric stimulation. The nerve conduction study of the left ulnar nerve revealed a sensorimotor conduction block of marked degree at a non-entrapment site, on the mid-upper arm (Fig. 2). F-wave latency of the right median nerve was significantly increased. Axonal loss was minimal. Based on the clinical and electrophysiological data, the diagnosis of MADSAM polyneuropathy was established.

Ultrasound examination was carried out five days later. A focal enlargement with an abnormal echotexture (hypoechogenity and loss of normal fascicular pattern) of the left ulnar nerve on the mid-upper arm, $13 \mathrm{~cm}$ proximal to the medial epicondyle was seen, corresponding exactly to the site of conduction block. On the left peroneal nerve, where the electrophysiological examination failed to localize the exact site of conduction block, ultrasound examination revealed a focal nerve enlargement with abnormal echotexture of the nerve just proximal to the most proximal site of electric stimulation, in the proximal part of the popliteal fossa (Fig. 2). There were mild enlargements and loss of normal fascicular structure on the median nerves on the upper arm on both sides.

\section{Discussion}

Our study is the first combined and detailed electrophysiologic and ultrasonographic evaluation of patients with MADSAM neuropathy. Striking concordance of electrophysiologic and ultrasonographic (morphologic) findings was found: marked focal enlargement and change of echostructure of the nerves were co-localized with existing conduction blocks of nerves clinically affected. Other segments of the affected nerves were normal. Milder focal ultrasonographic abnormalities were seen on nerves showing subclinical 
electrophysiological involvement. These findings reinforce the multifocal rather than the diffuse nature of the disease. Moreover, our study highlights how ultrasonography may help in localizing the pathological nerve segment when it is at a site inaccessible to electric stimulation, as seen with the peroneal nerve palsy in our second case, or when substantial secondary axonal loss precludes electrophysiological localization of the block, as with the radial nerve palsy in our first case. A novel and remarkable finding of our study is the detection of persistent ultrasonographic findings (focal enlargement and change of echostructure of the nerve) on nerves which have shown complete clinical and electrophysiological recovery following treatment, at sites precisely corresponding to the previous conduction blocks. Over a year has elapsed between recovery and the detection of these focal ultrasonographic abnormalities in our first case.

Previous studies have already described diffuse and/or focal sonographic enlargement of peripheral nerves and the brachial plexus in hereditary and acquired demyelinating neuropathies such as Charcot-Marie-Tooth-1A, CIDP, hereditary neuropathy with liability to pressure palsies (HNPP), and Guillain-Barré syndrome. ${ }^{2-4,6}$ It is unclear whether the presence or degree of nerve enlargement depends on the duration or treatment of disease. Beekman reported extensive multifocal nerve enlargement in patients with MMN. ${ }^{5}$ Among his patients, sonography showed abnormalities of clinically unaffected nerves or nerve segments without conduction blocks as well, however the separate analysis of patients with a short interval between electrodiagnostic and sonographic studies showed a consistent pattern. With respect to the analysis of sites of conduction blocks in demyelinating neuropathies and corresponding ultrasonographic findings, only a few cases of focal sonographic enlargements at sites of existing conduction blocks have been described, ${ }^{7,8,10}$ and there are no reports of ultrasonographic findings of nerve segments that show functional recovery. Ultrasonographic descriptions in MADSAM neuropathy are scarce, with only a single report in form of a 
$\operatorname{poster}^{10}$. Authors examined with ultrasound the median nerve in a patient with MADSAM neuropathy. Similarly to our results, they found a focal nerve enlargement corresponding to the site of conduction block but contrary to our findings, they described normalization of nerve size diameter parallel to the resolution of the conduction block. It is likely that the examined nerve was only mildly affected.

\section{Conclusion}

It is concluded that ultrasound examination in MADSAM neuropathy shows morphological abnormalities, focal enlargement and change of echostructure of the nerves, which correspond precisely to the sites of conduction blocks. This may be of significant help in localizing the pathological nerve segment when the conduction block is at an electrophysiologically inaccessible site or when substantial secondary axonal loss has occurred. Furthermore, these findings are also seen on nerves that have functionally recovered after treatment, allowing even retrospective confirmation of conduction block sites. It is postulated that these persisting morphological changes indicate foci of exaggerated remyelination. Further studies are needed to clarify the exact temporal relation of functional and morphological findings. 
Figure 1.

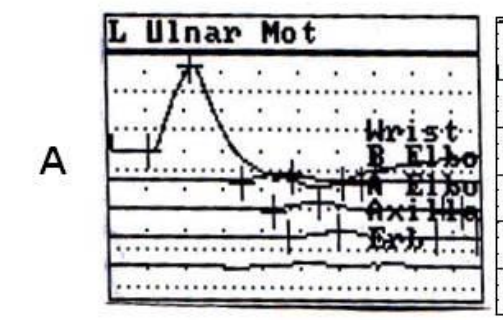

June 2009

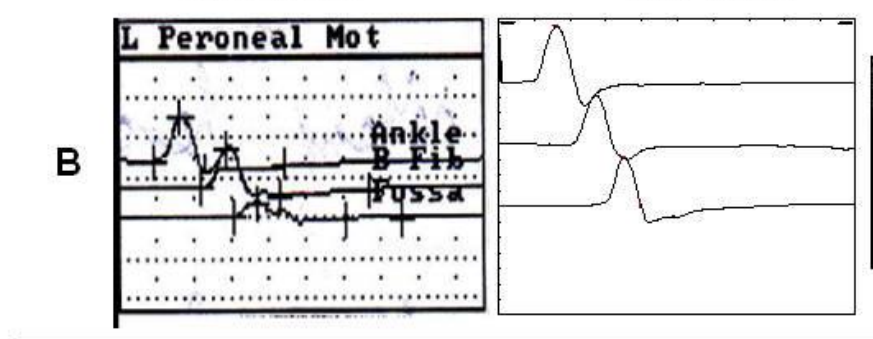

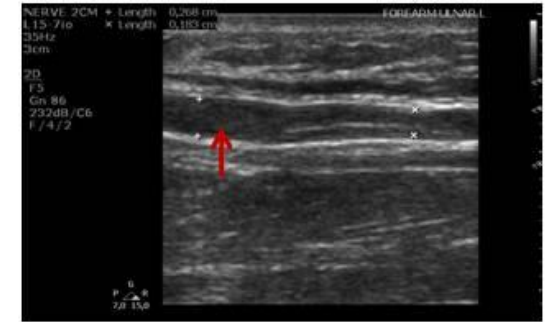

May 2011

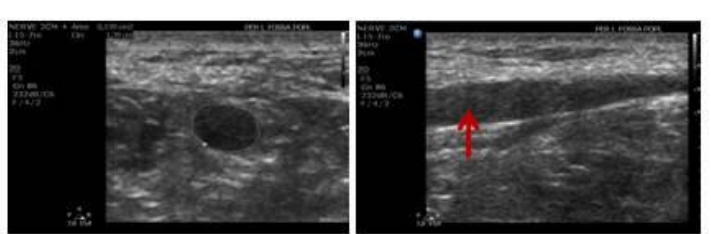

C
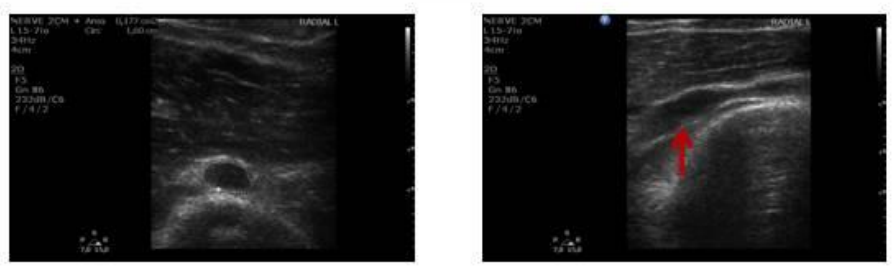

May 2011 

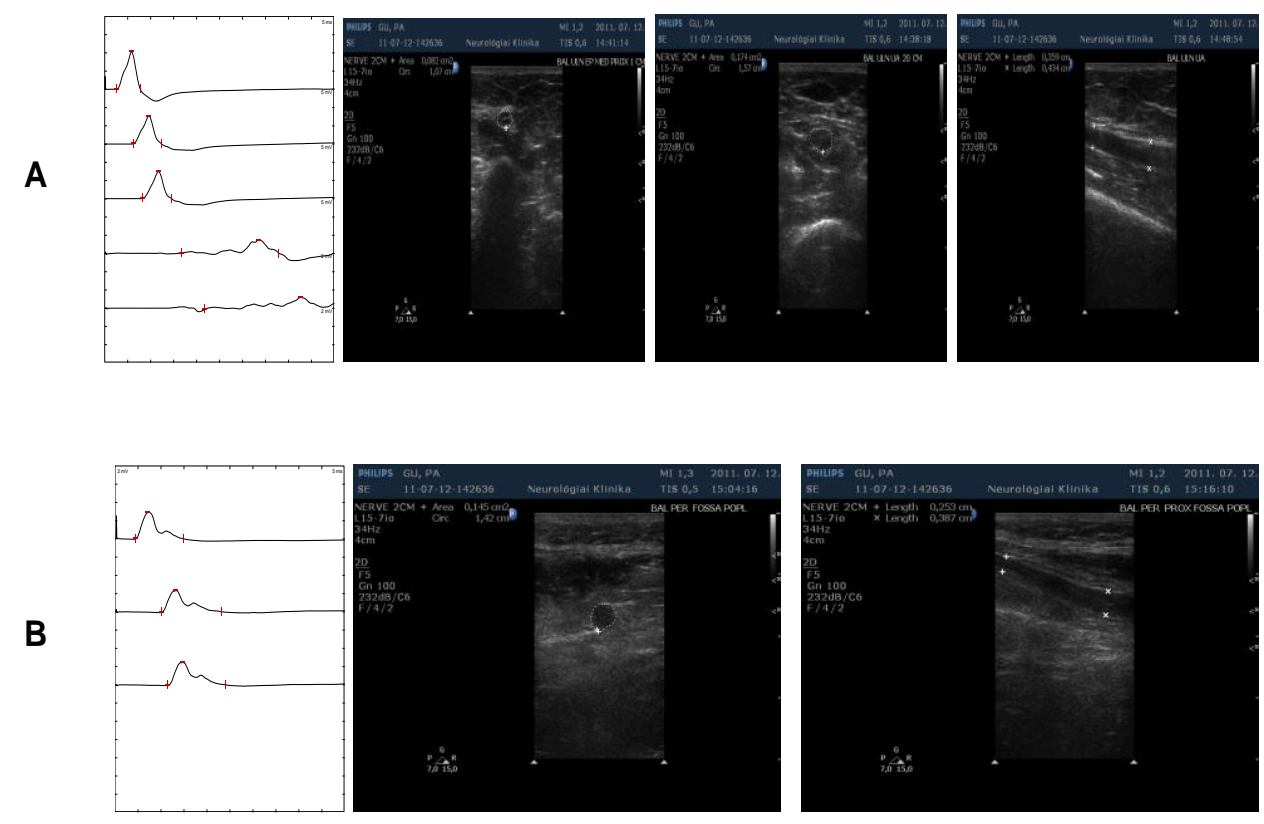

\section{Figure legends}

\section{Figure 1.}

A. Left ulnar nerve. The first nerve conduction study (May 2009) shows a conduction block on the forearm (scale/div: $5 \mathrm{mV}-3 \mathrm{~ms}$ ). The second nerve conduction study (May 2011) shows resolution of the conduction block (scale/div: $5 \mathrm{mV}-5 \mathrm{~ms}$ ). In both studies, the recording site was the abductor digiti minimi muscle, and the stimulation sites were the wrist, below elbow, above elbow, upper arm, and Erb point. Ultrasound (May 2011) shows focal enlargement of the nerve on the forearm (arrow), corresponding to the site of the previous conduction block: the nerve diameter on longitudinal scan increases from $1.8 \mathrm{~mm}$ to $2.6 \mathrm{~mm}$. The CSA of the nerve on transverse scan is $11.2 \mathrm{~mm}^{2}$ at $13 \mathrm{~cm}$ above the styloid process of the ulna and $5.9 \mathrm{~mm}^{2}$ at the proximal third of the forearm. 
B. Left peroneal nerve. The first nerve conduction study (May 2009) shows a conduction block at the fibular head or above (scale/div: $2 \mathrm{mV}-5 \mathrm{~ms}$ ). The second nerve conduction study (May 2011) shows resolution of the conduction block (scale/div: $2 \mathrm{mV}-5 \mathrm{~ms}$ ). In both studies, the recording site was the extensor digitorum brevis muscle, and the stimulation sites were the ankle, below fibular head, and popliteal fossa. Ultrasound (May 2011) shows focal enlargement of the nerve in the distal part of the popliteal fossa, corresponding to the site of the previous conduction block: the CSA of the nerve on transverse scan is $13.9 \mathrm{~mm}^{2}$ (11.6 $\mathrm{mm}^{2}$ at the fibular head), and the nerve is hypoechoic, its fascicular structure is no longer discerned. The same focal enlargement (arrow) is also shown on a longitudinal scan. C. Left radial nerve. Focal, spindle-like swelling (arrow) of the nerve in the spiral groove shown in transverse and longitudinal scans. On transverse scan, the CSA is significantly enlarged : $9 \mathrm{~mm}^{2}\left(3.5 \mathrm{~mm}^{2}\right.$ at the same level on the right side) and the echostructure of the nerve is pathological.

\section{Figure 2.}

A. Left ulnar nerve. The nerve conduction study shows a conduction block on the upper arm (scale/div: $5 \mathrm{mV}-5 \mathrm{~ms}$ ). The recording site was the abductor digiti minimi muscle, and the stimulation sites were the wrist, below elbow, above elbow, upper arm and Erb point. Ultrasound shows focal enlargement of the nerve on the upper arm corresponding to the site of the conduction block. CSA on transverse scan $1 \mathrm{~cm}$ above the medial epicondyle is $8 \mathrm{~mm}^{2}$, $13 \mathrm{~cm}$ proximal to the medial epicondyle is $17.5 \mathrm{~mm}^{2}$, and nerve diameter on longitudinal scan increases from $3.5 \mathrm{~mm}$ to $4.3 \mathrm{~mm}$ in this region (middle upper arm). The echostucture of the nerve is pathological. 
B. Left peroneal nerve. The nerve conduction study is normal (scale/div: $2 \mathrm{mV}-5 \mathrm{~ms}$ ). The recording site was the extensor digitorum brevis muscle, and the stimulation sites were the ankle, below fibular head, and popliteal fossa. Ultrasound shows focal enlargement of the nerve in the proximal part of the popliteal fossa: the CSA on transverse scan is $14 \mathrm{~mm}^{2}$ $\left(11 \mathrm{~mm}^{2}\right.$ at the fibular head), the nerve diameter on longitudinal scan increases from $2.5 \mathrm{~mm}$ to $3.8 \mathrm{~mm}$.

\section{Abbreviations:}

CIDP

CSA

HNPP

IVIG

MADSAM

MMN chronic inflammatory demyelinating polyneuropathy

cross sectional area hereditary neuropathy with liability to pressure palsies intravenous immunoglobulin treatment multifocal acquired demyelinating sensory and motor neuropathy multifocal motor neuropathy 


\section{References}

1. Beekman R, Visser LH. High resolution sonography of the peripheral nervous system- a review of the literature. Eur J Neurol 2004; 11(5):305-14.

2. Matsuoka N, Kohriyama T, Ochi K, Nishitani M, Sueda Y, Mimori Y, Nakamura S, Matsumoto M. Detection of cervical nerve root hypertrophy by ultrasonography in chronic inflammatory demyelinating polyradiculoneuropathy. J Neurol Sci 2004; 219(1-2):15-21.

3. Cartwright MS, Brown ME, Eulitt P, Walker FO, Lawson VH, Caress JB. Diagnostic nerve ultrasound in Charcot-Marie-Tooth disease type 1B. Muscle Nerve 2009; 40(1): 98-102.

4. Beekman R, Visser LH. Sonographic detection of diffuse peripheral nerve enlargement in hereditary neuropathy with liability to pressure palsies. J Clin Ultrasound 2002; 30(7):433-6.

5. Beekman R. Ultrasonography shows extensive nerve enlargement in multifocal motor neuropathy. Neurology 2005; 65:305-7.

6. Zaidman CM, Al-Lozi M, Pestronk A. Peripheral nerve size in normals and patients with polyneuropathy: an ultrasound study. Muscle Nerve 2009; 40(6): 960-6.

7. Granata G, Pazzaglia C, Canadro P, Luigetti M, Martinoli C, Sabatelli M, Padua L. Ultrasound visualisation of nerve morphological alteration at the site of conduction block. Muscle Nerve 2009; 40(6):1068-70.

8. Imamura K, Tajiri Y, Kowa H, Nakashima K. Peripheral nerve hypertrophy in chronic inflammatory demyelinating polyradiculoneuropathy detected by ultrasonography. Intern Med 2009; 48:581-582.

9. Sander H W, Latov N. Research criteria for defining patients with CIDP. Neurology 2003; 60(Suppl 3):S8-S15. 
10. Smith EC, Hibson,Webb LD, Massey E. Nerve ultrasound in motor conduction block: pre-and posttreatment finding. Muscle Nerve 2008; 38:1369. 